\title{
Research Highlight: A Rate-jump Method for Characterization of Soft Tissues using Nanoindentation techniques
}

\author{
B. Tang ${ }^{1}$ and A.H.W. Ngan ${ }^{2, *}$ \\ ${ }^{1}$ Department of Mechanical Engineering, \\ King Abdullah University of Science and Technology, Thuwal, Saudi Arabia \\ ${ }^{2}$ Department of Mechanical Engineering, \\ University of Hong Kong, Pokfulam Road, Hong Kong, P.R. China \\ *Corresponding Author (email: hwngan@hku.hk)
}

\begin{abstract}
The biomechanical properties of soft tissues play an important role in their normal physiological and physical function, and may possibly relate to certain diseases. The advent of nanomechanical testing techniques, such as atomic force microscopy (AFM), nano-indentation and optical tweezers, enables the nano/micro mechanical properties of soft tissues to be investigated, but in spite of the fact that biological tissues are highly viscoelastic, traditional elastic contact theory has been routinely used to analyze experimental data. In this article, a novel rate-jump protocol for treating viscoelasticity in nanomechanical data analysis is described.
\end{abstract}

\section{Introduction}

The mechanical behaviours of biological tissues are closely related to their physical and biological functions, in physiological or disease states. A better understanding of the mechanics of biological tissues can help understand the pathologenesis of diseases, developing new devices for treatment, and fabricating new biomaterials for tissue replacement [1-8]. In recent years there has been a lot of interest in applying nanomechanical characterization techniques with displacement and force resolutions in the nano-regime, including AFM, nanoindentation, optical trap, micro-pipette aspiration, and so forth, to characterize the mechanical and other physical behaviour of biological tissues [9-21]. Recent results indicate that certain types of diseases are intricately linked to the mechanical behaviour of the relevant cells and other, often nanoscale, protein building blocks of life [22-24]. Well known examples include breast cancer, where it is 
found that cancer cells are mechanically softer than normal cells [25], and malaria, where infected red blood cells are mechanically stiffer [12]. During cancer metastasis, the migration capability of tumor cells is essential for them to invade new organs, and so the reduced rigidity of cancerous cells could be a factor leading to their lack of contact inhibition, as well as increased penetration ability through membranes and blood vessel walls. It is therefore likely that a link exists between the spread of cancer cells through the blood stream or the lymphatic system, and the reduced mechanical rigidity of the cancerous cells. Other examples are also commonly found in disorders of human connective tissues such as bone and cartilage, and dental tissues such as enamel and dentine. For instance, the developmental defect of "molar incisor hypomineralization", commonly seen in the enamel of the first permanent molar and incisors, has been found to be associated with a reduction in the mechanical strength of not the micronsized enamel prisms themselves but the nanometric wide sheaths between them [26]. The mechanical integrity of tissue interfaces is also known to be important in understanding dysfunctions of connective tissues such as cartilage [27], and the nanomechanical properties of cartilage can also be used as an indicator to detect arthritis [28].

Testing biological tissues of nanometric volumes is a lot more challenging than testing synthetic materials in bulk scale primarily because of three issues, namely, (i) the test should resemble in vivo conditions which are usually wet and warm, (ii) biological tissues are usually highly viscoelastic, and (iii) a large scatter in data is the norm rather than exception because of the hierarchical structure of the tissues. These issues in particular affect whether intrinsic properties independent of the test conditions can be extracted from the test data. The wetness means not only that proper multi-phasic models need to be developed in order to extract intrinsic properties from the measured data [29], but liquid surface tension effects, which usually become significant or even dominating for micron- or nano-scale probe-sample contacts, need to be properly accounted for in the data analysis. Viscoelasticity becomes an issue because the existing data analysis protocols for nanomechanical measurements are based on the Hertzian theory or Sneddon contact theory which assumes pure elasticity. Although sophisticated viscoelastic models exist, it is surprising to see that most nanomechanical data published to-date from biological samples were obtained using the Hertzian model [2, 30-32]. In this highlight, we will focus on the viscoelastic effects during the micro/nano indentation measurement on soft tissues, and a simple and effective protocol to correct for the viscoelastic effects during nanomechanical tests.

\section{Viscoelastic effects during nanoindentation}

The viscoelastic deformation of soft tissues during mechanical testing can be used to extract parameters in a specific viscoelasticity model [33-37]. In such a model, the (viscous) dashpot elements correspond to permanent slippage or dissipative events of the interatomic bonds in the solid, and the (elastic) spring elements correspond to their stiffness during 
conservative stretching. Accurate extraction of both the elastic and viscous components of the viscoelastic network is therefore highly desirable. However, insofar as the elastic component is concerned, it has long been recognized that the accuracy of its determination with the nanoindentation technique may be severely affected by the viscous component of the deformation [5, 6, 20, 38-45], and simple corrective measures which may work for harder materials, such as increasing the holding before the unload or increasing the unloading rate, may not be effective for very soft materials $[40,41,44,46]$. Dynamic indentation with an oscillatory load superimposed on the basic load is routinely carried out, but the resultant storage and loss moduli are often found to depend on the oscillation frequency, rather than intrinsic material constants $[47,48]$.

A recent "rate-jump" method has been proven to be capable of returning an intrinsic elastic modulus that is independent of the test conditions on soft samples, and this can be easily adapted in different nanomechanical test platforms $[46,49]$. The key assumption is very mild an intrinsic constitutive law composing of any network arrangement of (in general) non-linear dashpots and linear elastic springs is assumed to hold within a very short time window $\left[t_{c}{ }^{-}, t_{\mathrm{c}}{ }^{+}\right]$ about time $t_{\mathrm{c}}$, at which a sudden step change in the loading rate is applied on the sample. Since the dashpots are described by relations of the form $\dot{\varepsilon}_{i j}=\dot{\varepsilon}_{i j}\left(\sigma_{k l}\right)$, the step change $\Delta \dot{\sigma}_{i j}$ in the stress rate field $\dot{\sigma}_{k l}$ at $t_{\mathrm{c}}$ does not result in any corresponding change in the strain rate field $\dot{\varepsilon}_{i j}$ across the dashpots, but because the linear elastic springs are described by relations of the form $\varepsilon_{i j}=s_{i j k l} \sigma_{k l}$ where $s_{i j k l}$ are elastic compliances, a step change $\Delta \dot{\varepsilon}_{i j}$ across the springs will occur according to

$$
\Delta \dot{\varepsilon}_{i j}=s_{i j k l} \Delta \dot{\sigma}_{k l}
$$

which is also the overall change for the sample. Eqn. (1) indicates that the fields $\Delta \dot{\sigma}_{i j}$ and $\Delta \dot{\varepsilon}_{i j}$ can be solved as a linear elastic problem with the elastic spring elements in the original viscoelastic network model while the dashpot elements are ignored [46]. The solution for a given test geometry is a linear relation between the step changes in the load and displacement rates at $t_{\mathrm{c}}$, with the linking proportionality constant being a lumped value of the elastic constants in the original viscoelastic model. Fitting such a relation to experimental results allows this lumped value to be measured as an intrinsic elastic modulus of the material.

\section{Rate-jump method in depth-sensing nanoindentation}

In depth-sensing nanoindentation, the elastic modulus and hardness are evaluated at the onset of an unloading stage following a load-hold stage [50]. Such an onset point for unloading is a rate-jump point, and solving eqn. (1) across this [46] gives rise to 
$\frac{1}{S_{e}}=\frac{1}{2 E_{r} a}=\frac{\Delta \dot{h}}{\Delta \dot{P}}=\frac{1}{S}-\frac{\dot{h}_{h}}{\dot{P}_{u}}$,

where $S=\mathrm{d} P / \mathrm{d} h$ ( $P=$ force, $h=$ displacement $)$ is the apparent tip-sample contact stiffness at the onset of unload, $\dot{h}_{h}$ is the displacement rate just before the unload, $\dot{P}_{u}$ is the unloading rate, and $S_{e}$ is the true (i.e. viscosity-corrected) tip-sample contact stiffness which is related to the reduced modulus $E_{r}$ and the tip-sample contact size $a$ by the Sneddon relation $S_{e}=2 E_{r} a$. The contact size $a$ can be estimated from a pre-calibrated shape function $f\left(h_{\mathrm{c}}\right)=\pi a^{2}$ of the tip, where the contact depth $h_{c}$ is obtainable using the Oliver-Pharr relation [50] with the apparent contact stiffness $S$ replaced by the true stiffness $S_{e}$ [41]:

$$
h_{c}=h_{\max }-\frac{P_{\max }}{S_{e}}=h_{\max }-P_{\max }\left(\frac{1}{S}-\frac{\dot{h}_{h}}{\dot{P}_{u}}\right) \text {. }
$$

Figure 1 shows the elastic modulus of mice cortical bone analyzed with the rate-jump method and the Oliver-Pharr method respectively [51]. Here, a multi-cycle loading schedule was used in which the elastic modulus was evaluated at the onset of each unloading cycle. In the earlier cycles, the elastic modulus returned by the Oliver-Pharr method is negative due to very severe viscoelastic effects. The modulus values measured with the rate-jump method are always positive and much more consistent.

When testing samples immersed in liquid in order to simulate in vivo conditions, the tip will be subjected to additional surface-tension forces which may need special considerations. Some commercial nanoindenters may allow tips attached to long shafts to be used and in such a situation, as long as the liquid surface stays within the straight part of the tip's shaft, the surfacetension force on the tip would be a constant. However, if the liquid level is within the sloping part of the tip, then the surface-tension force will vary as the tip travels up or down. Figure 2 shows the load-displacement curve of a nanoindentation test carried out on a rat cornea sample with a Berkovich indenter [52]. Here the sample was just barely, instead of fully, covered by a Dulbecco's Modified Eagle's liquid medium in order to simulate the in vivo conditions of cornea. Figure 2 shows that as the tip approached the solid surface, the tip first felt an attractive force, due to surface tension of the liquid, and when the tip penetrated deep enough to interact with the solid cornea, the load stopped decreasing and started to rise. The liquid level was likely to be within the sloping part of the tip, and since the Berkovich tip has a self-similar geometry, the liquid force can be modeled to be proportional to the tip displacement $h$, and the proportionality constant $K$ is simply the initial negative slope of the load-displacement curve [39]. The loaddisplacement curve can therefore be modeled by

$$
P=A\left(h-h_{a}\right)^{3 / 2}-K h
$$


where $A, h_{a}$ and $K$ are fitting parameters, and the true elastic stiffness can be calculated from the data just before and after the unloading point using the following equation [39]

$$
\frac{1}{S_{e}-K}=\left(\frac{1}{S}-\frac{\dot{h}_{h}}{\dot{P}_{u}}\right) \frac{1}{\left(1-\dot{P}_{h} / \dot{P}_{u}\right)} .
$$

\section{Rate-jump method in AFM nanoindentation}

Nanoindentation is also routinely carried out in commercial AFMs, but compared with using a commercial depth-sensing nanoindentation machine, more challenges arise in the AFM. Apart from the difficulty in accurately determining the tip-sample contact point in AFM nanoindentation [53, 54], unlike the diamond Berkovich tips usually used in depth-sensing nanoindentation, AFM tips are much sharper and more fragile, yet their accurate calibration is essential for reliable measurements. The usual tip shape calibration for a Berkovich diamond is unsuitable for AFM tips since the many indentations involved in obtaining the $f\left(h_{\mathrm{c}}\right)$ function (see eqn. (3) above) from a known sample (e.g. fused quartz) may already damage the tip. Furthermore, as is similar to depth-sensing nanoindentation, the commonly used data analysis protocol for AFM nanoindentation is also based on elastic contact theory - in this case the Hertzian law $P \propto E_{r} h^{3 / 2}$ for the ramping part of the load schedule, and this is equally problematic for soft biological tissues.

Based on eqn. (1), a rate-jump protocol for elastic modulus measurement from soft samples using AFM nanoindentation, in which the various problems discussed above are circumvented, was recently developed [49]. To avoid the tip-shape calibration, a flat-ended tip is used (Figure 3(a)), and this can be easily made from a commercial AFM tip by machining, e.g. by focused-ion-beam milling. The tip-sample contact size $a$ then remains constant for different indentation depths and this can be obtained easily by imaging of the tip in an electron microscope. By analyzing the mechanics of the AFM-cantilever-tip-sample interactions [49], application of eqn. (1) leads to the following relation:

$$
\frac{\Delta \dot{\delta}}{\Delta \dot{D}}=A\left(1+\frac{\alpha}{E_{r}}\right)
$$

where $\Delta \dot{\delta}$ an imposed step change in the rate of the PZT movement $\delta$ is of the sample base (i.e. the input), $\Delta \dot{D}$ the resultant step change in the rate of the photodiode signal $D$ due to the cantilever deflection (i.e. the output), $A$ the photo-diode sensitivity (i.e. cantilever deflection per unit sensor current generated), and $\alpha=k / 2 a$ is a cantilever-tip constant where $k$ is the force constant of the AFM-cantilever and $a$ the radius of the tip's end. In eqn. (6), only two constants, $A$ and $\alpha$, need to be calibrated each time a new tip is used, and these can be obtained by 
performing single indentations on two samples with known $E_{r}$ values. This amount of calibration is thought to be the minimum required, and furthermore, accurate determination of the initial contact point is not necessary because the contact size $a$ is constant due to the flat-ended shape of the tip. With $A$ and $\alpha$ known, eqn. (6) can be used to evaluate the $E_{r}$ of an unknown specimen, by measuring the $\Delta \dot{D}$ for a step change $\Delta \dot{\delta}$ imposed at some point during the load schedule. Figure 3(c-d) shows the elastic moduli measured from a UM1 oral cancer cell line shown in Figure 3(b) [55]. With the conventional Hertzian protocol, the measured modulus increases with the loading rate as a result of severe viscoelasticity of the cells. However, with the rate-jump protocol, the

measured modulus does not depend on the magnitude of the $\Delta \dot{\delta}$ used, and so it should be an intrinsic constant of the sample.

\section{Implementation in other platforms}

Apart from the nanoindentation platforms, the method has also been adapted for a microscale glass-plate compression platform in a micro-pipette system for characterizing the stiffness changes in collagen micro-masses inserted with human stem cells, as the latter differentiate into cartilage-like tissues [57]. The working principles of such a compression platform are similar to an AFM except that the size is in millimeter scale: the glass plate acts as an elastic cantilever and its clamp base at one end is displaced while its free end compresses the sample. The rate jump equation

\section{Future perspective and conclusion:}

The recent advancements in nanomechanical characterization techniques for soft materials provide a perspective for more systematic study on the links between diseases and the biomechanics of the relevant nano-scale building blocks of life. For example, cell motility is known to be driven by actin polymerization [56] - essentially, the unidirectional growth of actin filaments generates pico-Newton scale forces that lead to the movement of cells - and so a precise knowledge of the mechanics involved in the remodeling of actin network will likely be one of the keys to finding ways to limit the moving capability of cancer cells and hence reduce the risk of metastasis. At present, the hardware of these machines is capable of providing the nano- or even pico-scale resolution of forces and displacements, but key challenges remain in terms of how to deconvolve intrinsic properties from liquid and other effects in the measurement. Fluctuations are also important in such nano-scale systems, and the intrinsic behaviour needs to be separated from machine-driven noises. By the nature of eqn. (1), slow machine drifts will be subtracted out between the displacement data immediately before and after the rate-jump, and so the method should yield results that are free of influence of such drifts. When using eqn. (2) or (6) to obtain the step change in the response, data within time windows just before and just after the step change are curve-fitted to extrapolate back to the step-change point, and as a matter of 
good practice, it is always desirable to know the frequency of the machine noise so as to make sure that the time windows used for curve fitting are significantly wider.

The rate-jump protocol highlighted here provides an easy way of obtaining intrinsic elastic modulus of small volumes of soft materials in different nanomechanical platforms, without the need for equipment modification. Other platforms which can be utilized may include optical tweezers, in which nanomechanical work can also be carried out. As mentioned above, data interpretation, rather than equipment hardware development, seems to be the bottleneck, and there is ample opportunity for development for realistic nano-biomechanics models, against which experimental data are interpreted. Multi-phasic and poro-viscoelastic models are among the suitable candidates.

Finally, while the rate-jump method enables the effective elastic modulus to be obtained as an intrinsic property of the sample, no information about the dashpot component can be known from this method. In essence, the dasphots in the network do not respond to the rate-jump and only the elastic springs do, and so the response of the rate-jump gives the lumped effect of the springs only, while the dashpots are still unknown since their effects are subtracted out. The viscous component still needs to be obtained by analyzing the load relaxation or creep response of the sample against a presumed constitutive model, or as the loss modulus by means of dynamic load oscillations.

\section{References}

1. Radmacher, M., IEEE Engineering in Medicine and Biology Magazine, 1997. 16(2): p. 47-57.

2. Vinckier, A. and G. Semenza, FEBS Letters, 1998. 430(1-2): p. 12-16.

3. Bowen, W.R., R.W. Lovitt, and C.J. Wright, Biotechnology Letters, 2000. 22(11): p. 893-903.

4. $\quad$ Frederix, P.T., et al., MRS Bulletin, 2004. 29(7): p. 449-455.

5. $\quad$ Ebenstein, D.M. and L.A. Pruitt, Nano Today, 2006. 1(3): p. 26-33.

6. Oyen, M.L. and A.J. Bushby, International Journal of Surface Science and Engineering, 2007. 1(2-3): p. 180-197.

7. Lin, D.C. and F. Horkay, Soft Matter, 2008. 4(4): p. 669-682.

8. Cao, G.X. and N. Chandra, Physical Review E, 2010. 81(2).

9. $\quad$ Smith, S.B., Y.J. Cui, and C. Bustamante, Science, 1996. 271(5250): p. 795-799.

10. Murayama, Y. and M. Sano, Journal of the Physical Society of Japan, 2001. 70(2): p. 345-348.

11. Murade, C.U., et al., Nucleic Acids Research, 2010. 38(10): p. 3423-3431.

12. Dao, M., C.T. Lim, and S. Suresh, Journal of the Mechanics and Physics of Solids, 2003. 51(11-12): p. 2259-2280.

13. Zhao, R.G., K.L. Sider, and C.A. Simmons, Acta Biomaterialia, 2011. 7(3): p. 12201227.

14. Henriksen, J.R. and J.H. Ipsen, European Physical Journal E, 2004. 14(2): p. 149-167. 
15. Sun, Y.L., et al., Journal of Biomechanics, 2004. 37(11): p. 1665-9.

16. Luo, Z.P., et al., Biorheology, 2004. 41(3-4): p. 247-54.

17. Lieber, S.C., et al., American Journal of Physiology-Heart and Circulatory Physiology, 2004. 287(2): p. H645-H651.

18. Na, S., et al., Biomechanics and Modeling in Mechanobiology, 2004. 3(2): p. 75-84.

19. Rico, F., et al., International Journal of Nanotechnology, 2005. 2(1-2): p. 180-194.

20. Ni, G.X., et al., Journal of Biomedical Materials Research Part A, 2007. 83A(2): p. 570576.

21. Ni, G.X., et al., Biomaterials, 2006. 27(9): p. 1963-1970.

22. Hammond, N.A. and R.D. Kamm, Biomaterials, 2008. 29(21): p. 3152-3160.

23. Power, R.E., et al., Journal of Urology, 2004. 171(1): p. 457-461.

24. Suresh, S., et al., Acta Biomaterialia, 2005. 1(1): p. 15-30.

25. Guck, J., et al., Biophysical Journal, 2005. 88(5): p. 3689-3698.

26. Chan, Y.L., A.H.W. Ngan, and N.M. King, 2010. 38(3): p. 237-244.

27. Oegema, T.R., et al., Microscopy Research and Technique, 1997. 37(4): p. 324-332.

28. Stolz, M., et al., Nature nanotechnology, 2009. 4(3): p. 186-92.

29. Mow, V.C., et al., Journal of Biomechanics, 1989. 22(8-9): p. 853-861.

30. Liang, X.M., G.Z. Mao, and K.Y.S. Ng, Journal of Colloid and Interface Science, 2004. 278(1): p. 53-62.

31. Rosenbluth, M.J., W.A. Lam, and D.A. Fletcher, Biophysical Journal, 2006. 90(8): p. 2994-3003.

32. Park, S., et al., Proceedings of the Institution of Mechanical Engineers Part H-Journal of Engineering in Medicine, 2009. 223(H3): p. 339-347.

33. Yang, G.W., et al., Journal of Colloid and Interface Science, 2006. 297(1): p. 104-111.

34. Svensson, R.B., et al., Journal of the Mechanical Behaviour of Biomedical Materials, 2010. 3(1): p. 112-115.

35. Attard, P., Journal of Physics-Condensed Matter, 2007. 19(47).

36. Oyen, M.L., Journal of Materials Research, 2005. 20(8): p. 2094-2100.

37. Cheng, L., et al., Mechanics of Materials, 2005. 37(1): p. 213-226.

38. Cheng, Y.T. and C.M. Cheng, Materials Science and Engineering a-Structural Materials Properties Microstructure and Processing, 2005. 409(1-2): p. 93-99.

39. Tang, B. and A.H.W. Ngan, Soft Materials, 2007. 5(4): p. 169-181.

40. Tang, B. and A.H.W. Ngan, Soft Materials, 2004. 2(2-3): p. 125-144.

41. Tang, B. and A.H.W. Ngan, Journal of Materials Research, 2003. 18(5): p. 1141-1148.

42. Franke, O., M. Goken, and A.M. Hodge, JOM, 2008. 60(6): p. 49-53.

43. Chudoba, T. and F. Richter, Surface \& Coatings Technology, 2001. 148(2-3): p. 191-198.

44. Fan, Z.F. and J.Y. Rho, Journal of Biomedical Materials Research Part A, 2003. 67A(1): p. 208-214.

45. Feng, G. and A.H.W. Ngan, Journal of Materials Research, 2002. 17(3): p. 660-668.

46. Ngan, A.H.W. and B. Tang, Journal of Materials Research, 2009. 24(3): p. 853-862.

47. Li, X.D. and B. Bhushan, Materials Characterization, 2002. 48(1): p. 11-36.

48. Mencik, J., et al., Journal of Materials Research, 2005. 20(10): p. 2660-2669.

49. Tang, B. and A.H.W. Ngan, Philosophical Magazine, 2011. 91(7-9): p. 1329-1338.

50. Oliver, W.C. and G.M. Pharr, Journal of Materials Research, 1992. 7(6): p. 1564-1583.

51. Tang, B., A.H.W. Ngan, and W.W. Lu, Journal of Materials Science-Materials in Medicine, 2007. 18(9): p. 1875-1881. 
52. Wu, K.S. et al., "Micro-scale Stiffness Change of Cornea Tissues Suffered from Elevated Intraocular Pressure Investigated by Nanoindentation", accepted for publication in Soft Materials.

53. Dimitriadis, E.K., et al., Biophysical Journal, 2002. 82(5): p. 2798-2810.

54. Crick, S.L. and F.C.P. Yin, Biomechanics and Modeling in Mechanobiology, 2007. 6(3): p. 199-210.

55. Zhou, Z.L., et al., Journal of the Mechanical Behaviour of Biomedical Materials, 2012. 8: p. 134-142.

56. Theriot, J.A. and T.J. Mitchison, Nature, 1991. 352(6331): p. 126-131.

57. Chan, B.P., Li, C.H., Au-yeung, K.L., Sze, K.Y. and Ngan, A.H.W., Annals of Biomedical Engineering, 2008. 36: p. 1254-1267.

\section{Figure Captions}

Figure 1. The elastic modulus of mice cortical bone analyzed with Oliver-Pharr method and ratejump method. The insert shows the identical multi-cycle loading schedule for all the tests, in which the elastic modulus was calculated at the onset of each unloading portion. Data from ref. [51].

Figure 2. Indentation load-displacement curve of rat cornea sample barely covered in a liquid medium by a Berkovich tip. Data from ref. [52].

Figure 3. (a) Flat-ended tip for AFM nanoindentation. (b) UM1 oral cancer cells in Dulbecco's Modified Eagle Medium: Nutrient Mixture F-12 (DMEM/F12) Medium. (c-d): Elastic modulus of UM1 cells measured with (c) Hertzian model and (d) rate jump method. Data from ref. [55]. 


\section{Figures:}

\section{Oliver-Pharr Method}

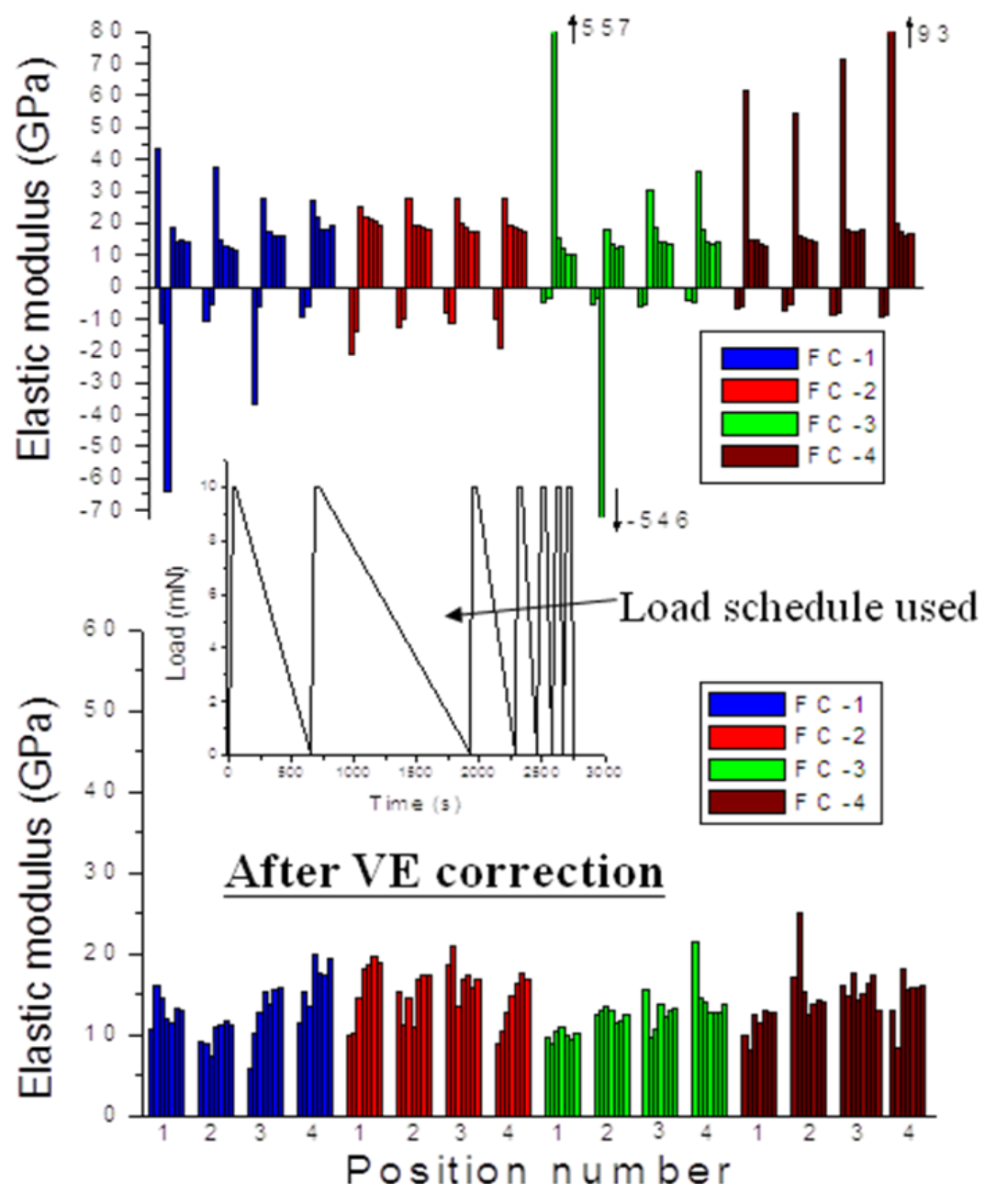

Figure 1. The elastic modulus of mice cortical bone analyzed with Oliver-Pharr method and ratejump method. The insert shows the identical multi-cycle loading schedule for all the tests, in which the elastic modulus was calculated at the onset of each unloading portion. Data from ref. [51]. 


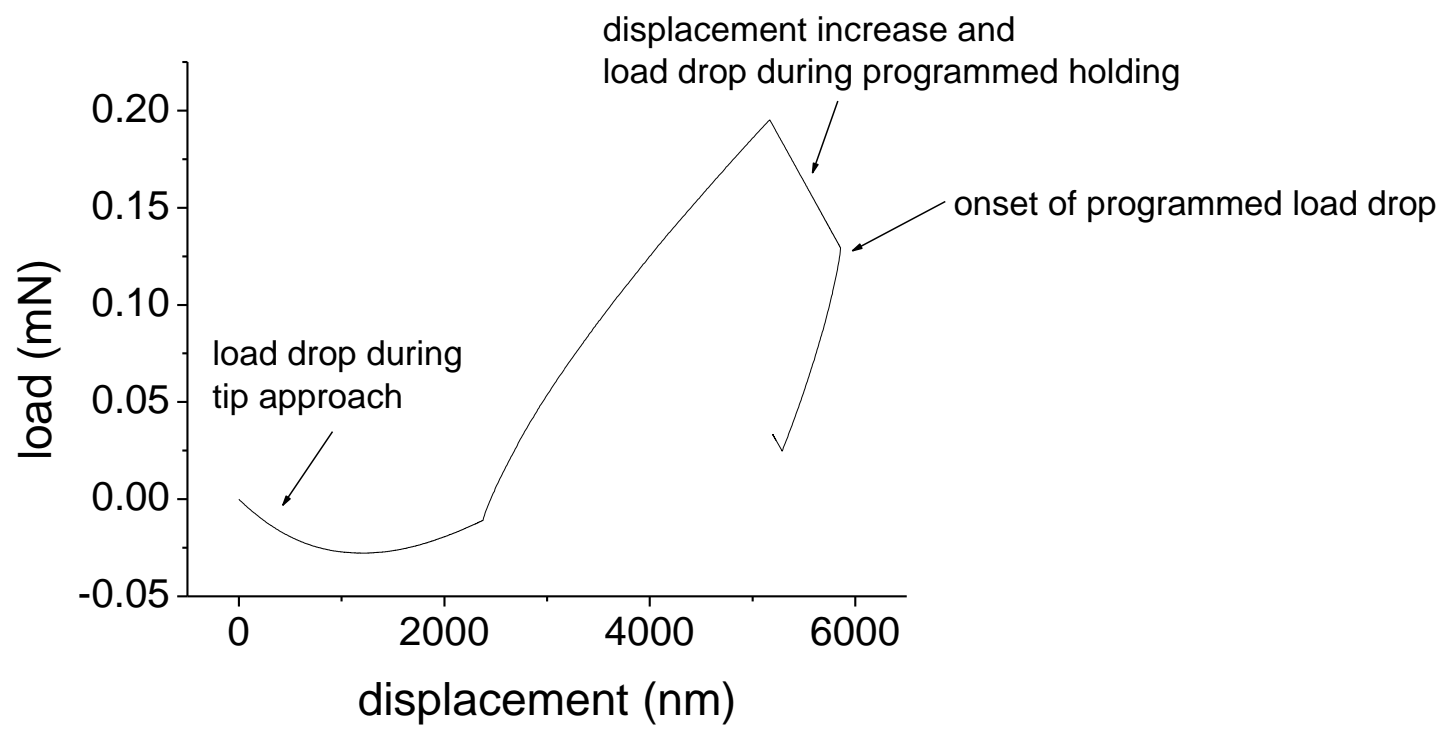

Figure 2. Indentation load-displacement curve of rat cornea sample barely covered in a liquid medium by a Berkovich tip. Data from ref. [52]. 


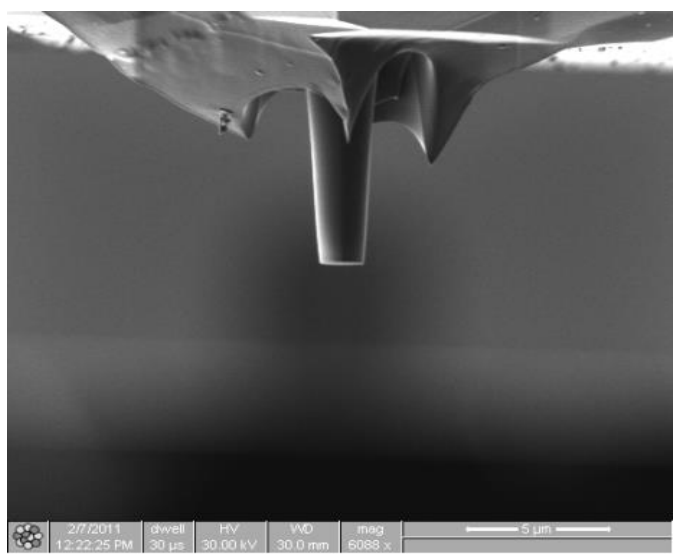

(a)

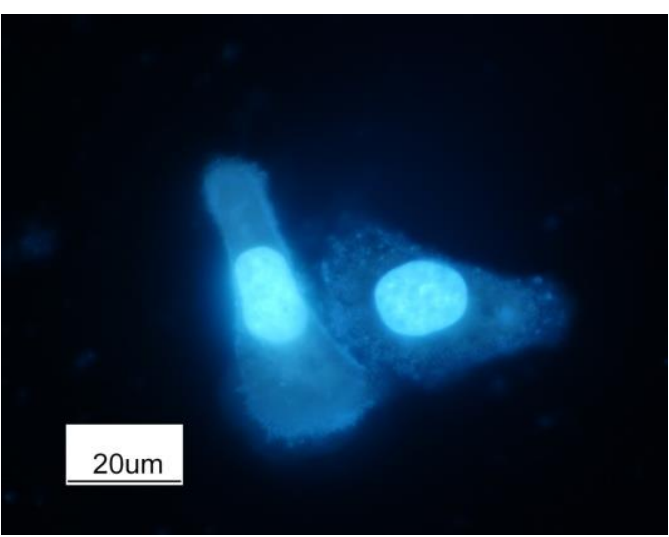

(b)

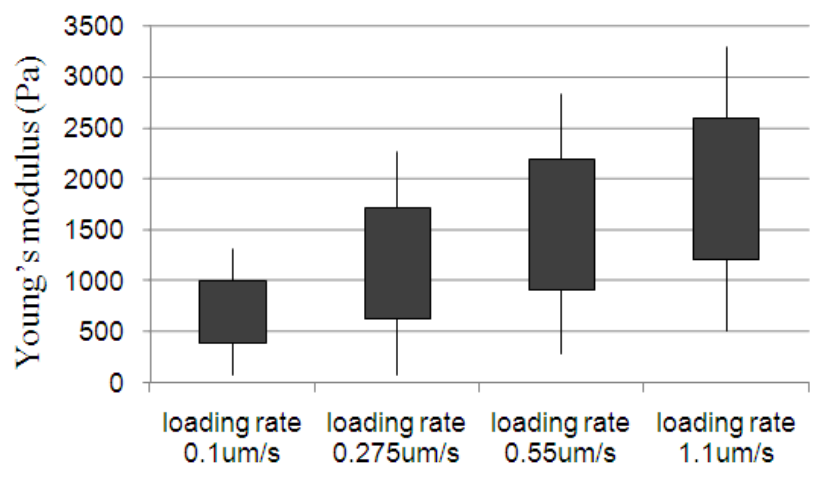

(c)

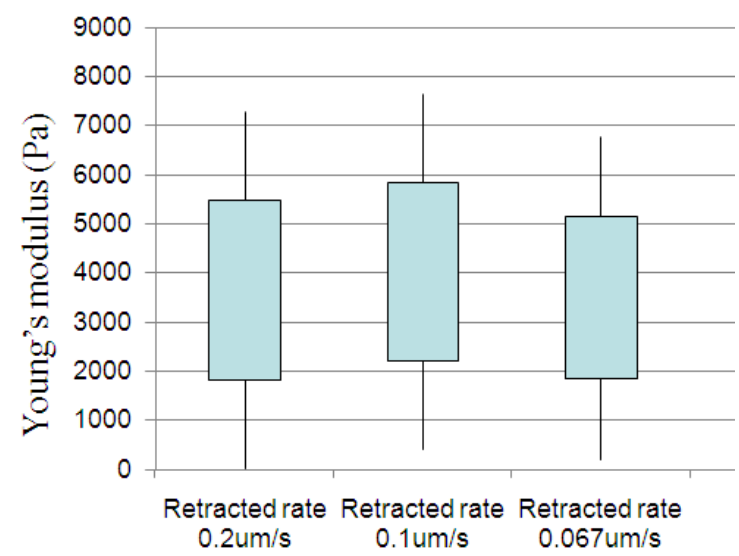

(d)

Figure 3. (a) Flat-ended tip for AFM nanoindentation. (b) UM1 oral cancer cells in Dulbecco's Modified Eagle Medium: Nutrient Mixture F-12 (DMEM/F12) Medium. (c-d): Elastic modulus of UM1 cells measured with (c) Hertzian model and (d) rate jump method. Data from ref. [55]. 